\title{
Outcomes of patients with refractory out-of-hospital cardiac arrest transported to an ECMO centre compared with transport to non-ECMO centres
}

Stephen A Bernard, Sarah J Hopkins, Jocasta C Ball, Dion A Stub, Michael W Stephenson, Vinodh B Nanjayya, Vincent A Pellegrino, Jayne Sheldrake, Alexander C Richardson and Karen L Smith

Out-of-hospital cardiac arrest (OHCA) is a common event associated with a high mortality rate. In emergency medical services (EMS) that provide advanced life support at scene, transport to an emergency department (ED) with ongoing cardiopulmonary resuscitation (CPR) in patients who do not achieve return of spontaneous circulation (ROSC) at scene is associated with low survival rates. ${ }^{1}$ However, in recent years, transport of the patient with refractory OHCA to hospital for extracorporeal membrane oxygenation (ECMO) during CPR (ECPR) has been shown to be associated with improved outcomes. ${ }^{2-4}$

The initial experience of ECPR at the ECMO centre in Melbourne, Australia, was supportive for the transport of patients with refractory OHCA to that centre. ${ }^{5}$ In that report, 11 patients with refractory OHCA were transported to the centre between 2012 and 2014, and ECMO was established in nine patients $(82 \%)$. Of these, three patients survived (33\%). Subsequently, in November 2015 all intensive care ambulances in Melbourne were equipped with an mCPR device (LUCAS 2, Stryker). The Ambulance Victoria clinical practice guideline was revised to recommend that selected patients with refractory OHCA should be transported to the ECPR centre if arrival at that centre within 45 minutes of arrest was possible.

In this study, we review the outcomes of patients in refractory arrest who were transported to the ECPR hospital and compare hospital and 12-month functional outcomes with patients transported to hospitals without ECPR capability between January 2016 and December 2019.

\section{Methods}

Ambulance Victoria provides the EMS for the state of Victoria, Australia. The city of Melbourne has a population of 5 million and an area of $10000 \mathrm{~km}^{2}$. The response to suspected OHCA in Melbourne is three-tiered and includes fire services, who provide basic life support and semiautomatic defibrillation; ambulance paramedics, who are authorised to insert a supraglottic airway and administer intravenous adrenaline; and intensive care paramedics,

\begin{abstract}
Objective: To compare the outcomes of patients with refractory out-of-hospital cardiac arrest (OHCA) transported to a hospital that provides extracorporeal membrane oxygenation (ECMO) during cardiopulmonary resuscitation (ECPR) with patients transported to hospitals without ECPR capability.
\end{abstract}

Design, setting: Retrospective review of patient care records in a pre-hospital and hospital setting.

Participants: Adult patients with OHCA who left the scene and arrived with cardiopulmonary resuscitation in progress at 16 hospitals in Melbourne, Australia, between January 2016 and December 2019.

Intervention: For selected patients transported to the ECPR centre, initiation of ECMO.

Main outcome measures: Survival to hospital discharge and 12-month quality of life.

Results: There were 223 eligible patients during the study period. Of 49 patients transported to the ECPR centre, 23 were commenced on ECMO. Of these, survival to hospital with good neurological recovery (Cerebral Performance Category [CPC] score 1/2) occurred in $4 / 23$ patients. Four other patients developed return of spontaneous circulation in the ECPR centre before cannulation of whom one survived, giving overall good functional outcome at 12 months survival of $5 / 49(10.2 \%)$. There were 174 patients transported to the 15 non-ECPR centres and 3/174 (2\%) had good functional outcome at 12 months. After adjustment for baseline differences, the odds ratio for good neurological outcome after transport to an ECPR centre compared with a non-ECPR centre was $4.63(95 \% \mathrm{Cl}, 0.97-$ 22.11; $P=0.055)$.

Conclusion: The survival rate of patients with refractory OHCA transported to an ECPR centre remains low. Outcomes in larger cities might be improved with shorter scene times and additional ECPR centres that would provide for earlier initiation of ECMO.

Crit Care Resusc 2022; 24 (1): 7-13 


\section{ORIGINAL ARTICLES}

who undertake intubation and also administer intravenous amiodarone for ventricular fibrillation/pulseless ventricular tachycardia (VF/pVT) that is refractory to defibrillation. The cardiac arrest protocols follow the Australian and New Zealand Council on Resuscitation guidelines. ${ }^{6}$

There are 16 hospitals in Melbourne with cardiac services that receive OHCA patients. One of these hospitals (the Alfred Hospital) is the designated state centre for heart and lung transplantation and has the capability of provision of ECPR for patients with refractory OHCA. During the study period, no other hospital in Victoria had the capability of providing ECPR to adult patients (aged $>15$ years) with refractory OHCA.

The Ambulance Victoria clinical practice guidelines for the management of refractory cardiac arrest recommended that the patients to be considered for transport with mCPR to the nearest appropriate ED were those with initial cardiac rhythm VF/pVT or pulseless electrical activity, patients with no major comorbidity, and patients with a bystander-witnessed arrest. ${ }^{7}$ If the patient was aged $<65$ years and arrival at the ECPR centre was possible within 45 minutes of the onset of arrest (or 60 minutes if the age was 16-35 years), then transport was undertaken to that centre, bypassing other EDs as necessary. The decision to transport from scene to hospital with MCPR was made by the paramedics at scene, taking into account extrication and transport time to hospital.

Patients who established ROSC at scene but re-arrested before hospital arrival or patients who departed the scene with $\mathrm{mCPR}$ but established ROSC during transport were not included in this study. Paediatric patients (aged $<16$ years) and patients with cardiac arrest due to trauma were also excluded.

The procedure for ECPR at this centre has been described previously ${ }^{5}$ and follows recommended guidelines. ${ }^{8}$ In summary, cannulation is undertaken by intensive care physicians in the ED using percutaneous needle, guidewire, dilation, or cannula insertion into the femoral vessels under ultrasound imaging. Once the access and return ECMO cannulae are inserted, blood flow via the oxygenator is commenced. When the patient is stabilised, consideration is then given to either transfer to the cardiac catheterisation laboratory for coronary angiography or transfer to a CT scanner if a non-cardiac event such as pulmonary embolism is considered to be the cause of the arrest. Of note, following publication of the clinical trial of a rapid intravenous infusion of $2 \mathrm{~L}$ of ice-cold saline during $C P R,{ }^{9}$ this treatment was not used as in the CHEER trial. ${ }^{5}$ Moreover, following the results of the TTM study in 2013, ${ }^{10}$ the target temperature for all OHCA patients at the ECPR centre during the study period was $36^{\circ} \mathrm{C}$ for the first 24 hours.
For this study, data were sourced from the Victorian Ambulance Cardiac Arrest Registry (VACAR) and the Alfred Hospital medical records for patients transported to that centre. The VACAR methodology has been described previously. ${ }^{11}$ This registry has pre-hospital data on all OHCA in Victoria and includes hospital discharge status along with 12-month telephone follow-up on adult survivors to assess quality of life using the Glasgow Outcome Scale-Extended (GOS-E), the 12-item Short Form Health Survey (SF-12) and the EuroQol (EQ-5D) health status instrument. ${ }^{12}$ On the basis of these, patients were allocated a Cerebral Performance Category (CPC) score 1/2 (normal/minimal disability) or 3/4/5 (moderate disability/severe disability/death).

The study period was January 2016 to December 2019, with the 12-month follow-up of adult survivors concluding in December 2020. The primary outcome measure for this study was the rate of good neurological outcome (CPC score 1/2) at 12 months for patients in refractory arrest transported to an ECPR centre compared with those transported to a non-ECPR centre.

\section{Statistical analyses}

Analyses were performed using Stata 15 (StataCorp, 2017, College Station, TX). Descriptive statistics are presented as frequencies and proportions for categorical data and median and interquartile ranges (IQRs) or mean and standard deviation (SD) for continuous variables. To compare outcomes between ECPR and non-ECPR hospitals, a $t$ test or Wilcoxon rank sum test (numerical values) and $\chi^{2}$ analysis or two-tailed Fisher exact test (categorical values) were performed. Logistical regression was based on baseline characteristics that were significantly different on univariate analysis (age, location, shockable rhythm and paramedic intubation) to calculate an adjusted odds ratio (OR) for outcomes.

\section{Ethics approval}

This study was approved by the Human Research Ethics Committee of the Alfred Hospital (54/19). The collection of data for VACAR was approved by the Monash University Human Research Ethics Committee (Approval No. 21046).

\section{Results}

Between January 2016 and December 2019, resuscitation was commenced in 12183 patients in Victoria. In 3944 patient $(32.4 \%)$, ROSC was achieved at scene or before arrival in the ED (Figure 1). There were 223 cardiac arrest patients (1.8\%) who were transported by ambulance and arrived at an ED in Victoria with $\mathrm{mCPR}$ in progress, with the majority of these patients $(78.0 \%)$ transported to a non-ECMO centre. There 
Figure 1. Incidence and initial outcomes of cardiac arrest patients during the study period

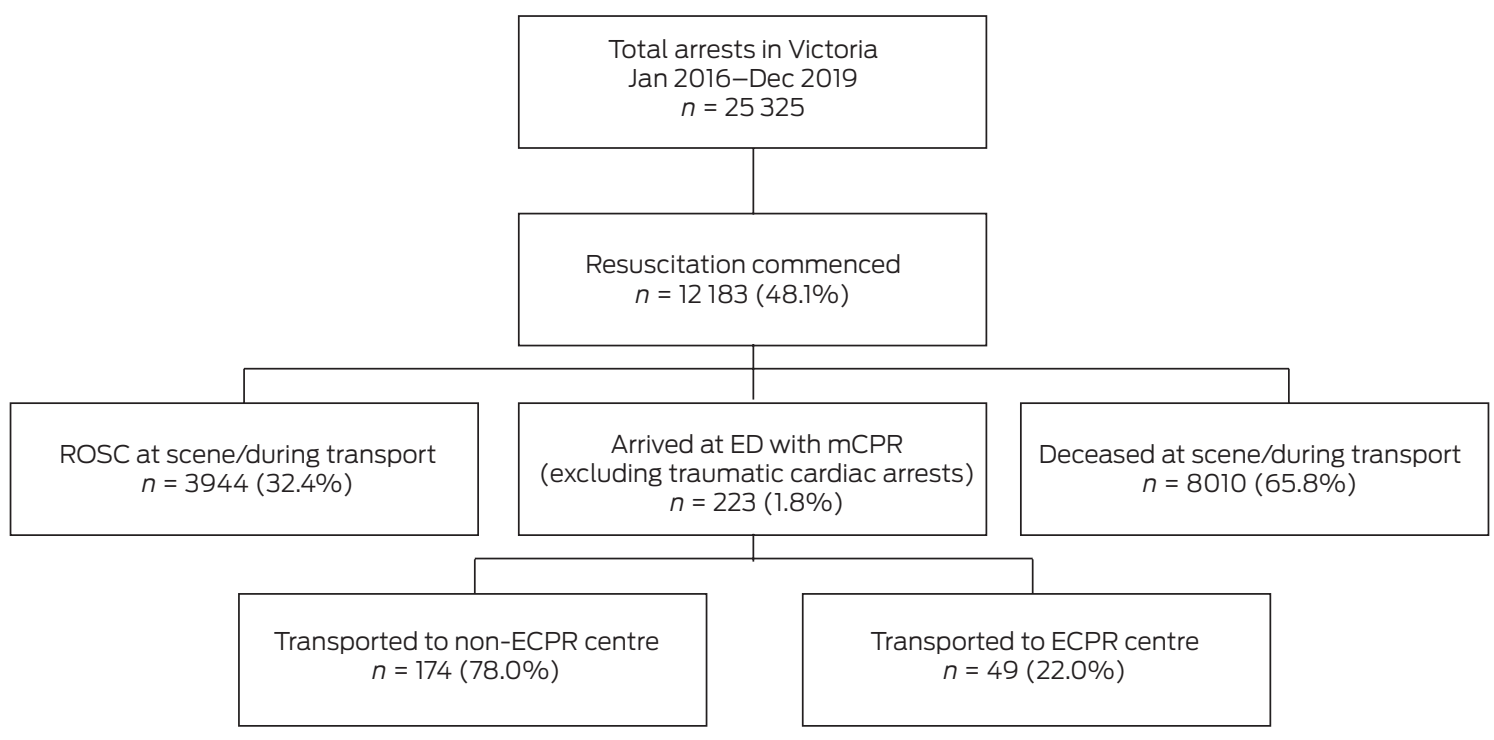

$E D=$ emergency department; $E C P R=$ extracorporeal membrane oxygenation assisted cardiopulmonary resuscitation; $m C P R=$ mechanical cardiopulmonary resuscitation; ROSC $=$ return of spontaneous circulation.

were six patients with hospital data not available. All patients transported with $\mathrm{mCPR}$ were aged $>15$ years.

Table 1 compares the baseline characteristics of patients who arrived at the ECPR centre with the non-ECPR centres. Of note, there were significant differences in the two groups for factors that are known to be associated with improved outcomes, such as age, site of arrest, shockable versus non-shockable initial cardiac rhythm, and cardiac arrest in a public place.

Of the 49 patients who arrived at the ECPR centre, the time from arrest onset to arrival at the ECPR centre was a median of 63.9 minutes (IQR, 54.3-82.0). In the ED, ECMO cannulation was attempted in 25 patients and established in 23 patients. In two patients, attempts at percutaneous femoral cannulation were unsuccessful and resuscitation was ceased. The time from commencement of the ECPR procedure to establishment of ECMO flow was not available from the electronic medical record.

Of the 23 patients in whom cannulation was successful and ECMO was commenced, four (17.4\%) survived to hospital discharge, all with CPC score $1 / 2$ at the 12-month follow-up (Table 2). Of the 19/23 patients who did not survive to hospital discharge, the causes of death were:

- in six of 19 patients, organ perfusion was not able to be achieved with ECMO, as shown by profound hypotension and increasing lactate levels despite fluid therapy and high dose pressor agents;
- in three of 19 patients, multi-organ system failure developed over the first 24 hours;

- in two of 19 patients, brain death developed over 36 hours;

- in two of 19 patients, major intra-abdominal bleeding occurred that led to the patient becoming unsupportable despite transfusion of blood products; and

- the remaining six of 19 patients had severe hypoxicischaemic neurological injury and had active medical treatment withdrawn between 5 and 10 days.

Of the 24 patients in whom ECMO was not attempted, four developed ROSC shortly after ED arrival and of these, one survived to hospital discharge. At 12-month followup, this patient had CPC score $1 / 2$. The reasons for not attempting ECMO in the remaining 20/24 patients were:

- CPR duration significantly exceeded 45 minutes in patients aged $>35$ years and thus was considered too prolonged (13/20);

- major significant comorbidities (three of 20);

- significant airway bleeding (two of 20); and

- age outside the eligibility criteria (two of 20).

The hospital and 12-month outcomes of the 174 patients transported to one of the 15 non-ECPR centres are shown in Table 2. The rate of ROSC in the ED and interventions such as angiography or computed tomography were not available for those patients. At the non-ECPR centres, seven $(4.0 \%)$ patients were discharged alive and, at 12-month 
Table 1. Baseline characteristics

\begin{tabular}{|c|c|c|c|}
\hline & ECPR centre & Non-ECPR centre & $P$ \\
\hline Total number of patients & 49 & 174 & \\
\hline Sex, male & $39(79.6 \%)$ & $135(77.6 \%)$ & 0.765 \\
\hline Age (years), mean (SD) & $46.4(13.3)$ & $54.5(16.0)$ & $<0.001$ \\
\hline Bystander-witnessed & $27(55.1 \%)$ & $88(50.6 \%)$ & 0.575 \\
\hline Bystander CPR & $30(61.2 \%)$ & $100(57.5 \%)$ & 0.638 \\
\hline Paramedic-witnessed & $13(26.5 \%)$ & $51(29.3 \%)$ & 0.704 \\
\hline Arrest at home & $20(40.8 \%)$ & $105(60.3 \%)$ & 0.015 \\
\hline Arrest at public place & $24(49.0 \%)$ & $49(28.2 \%)$ & 0.006 \\
\hline Cardiac aetiology & $44(89.8 \%)$ & $151(86.8 \%)$ & 0.574 \\
\hline Respiratory aetiology & $2(4.1 \%)$ & $10(5.8 \%)$ & 0.999 \\
\hline Other aetiology & $3(6.1 \%)$ & $13(7.5 \%)$ & 0.517 \\
\hline Initial rhythm VFNT & $35(71.4 \%)$ & $94(54.0 \%)$ & 0.029 \\
\hline Initial rhythm PEA & $9(18.4 \%)$ & $55(31.6 \%)$ & 0.070 \\
\hline Initial rhythm asystole & $4(8.2 \%)$ & $23(13.2 \%)$ & 0.459 \\
\hline Initial temperature $\left({ }^{\circ} \mathrm{C}\right)$, median $(\mathrm{IQR})$ & $35.8(34.5-36.5)$ & $35.6(34.5-36.2)$ & 0.532 \\
\hline Call time to EMS arrival (min), median (IQR) & $8.1(6.0-10.8)$ & $8.3(6.7-10.8)$ & 0.501 \\
\hline EMS arrival to EMS depart scene (min), median (IQR) & $41(31-52.5)$ & $45(35-56)$ & 0.200 \\
\hline Transport time (min), median (IQR) & $13(8.5-20)$ & $13(9-19)$ & 0.950 \\
\hline Call time to arrival in ED (min), median (IQR) & $63.9(54.3-82.0)$ & $67.8(57.0-82.2)$ & 0.344 \\
\hline Total bolus adrenaline dose (mg), mean (SD) & $10.3(4.6)$ & $10.9(4.4)$ & 0.214 \\
\hline Amiodarone given & $29(59.2 \%)$ & $97(55.8 \%)$ & 0.668 \\
\hline Defibrillation administered & $41(83.7 \%)$ & $122(70.1 \%)$ & 0.059 \\
\hline Intubation & $41(83.7 \%)$ & $163(93.7 \%)$ & 0.024 \\
\hline
\end{tabular}

$C P R=$ cardiopulmonary resuscitation; $E C P R=$ extracorporeal membrane oxygenation assisted cardiopulmonary resuscitation; ED = emergency department; EMS = emergency medical services; IQR = interquartile range; PEA = pulseless electrical activity; SD = standard deviation; VF/VT = ventricular fibrillation/ pulseless ventricular tachycardia.

follow up, three of these seven had a CPC score 1/2. The OR for 12-month survival with good neurological outcome after transport to an ECPR centre compared with a nonECPR centre was $4.83(95 \% \mathrm{Cl}, 0.98-25.17 ; P=0.013)$. However, after adjustment for baseline differences, the OR for good neurological outcome after transport to an ECPR centre compared with a non-ECPR centre was $4.63(95 \%$ $\mathrm{Cl}, 0.97-22.11 ; P=0.055)$.

\section{Discussion}

This study of patients with refractory cardiac arrest who were transported to an ED with $\mathrm{mCPR}$ in progress demonstrates that the rate of survival with good neurological outcome at 12 months is low. Survival is higher for patients who arrived at an ECPR centre and were established on ECMO, but it did not reach statistical significance.

An important finding from our study is that the times between cardiac arrest and arrival at the ECPR centre were longer than in some other reports. For example, Yannopoulos and colleagues ${ }^{2}$ in Minnesota, US, conducted a randomised trial comparing ECPR with continued advanced life support CPR in patients with refractory OHCA transported to their centre. There were 29 patients allocated to either continuing CPR only ( $n=15)$ or to ECPR $(n=14)$. Survival to hospital discharge was one of 15 CPR-only patients (7\%) versus six of 14 ECPR patients (43\%; risk difference, $36.2 \%$; posterior probability of ECMO superiority, 0.9861 ). Of note, in that study, the mean time from call to EMS arrival was 6 minutes, the scene time was 22.5 minutes, and transport time was 19 minutes, giving a total low flow time of 48 minutes before the start of ECMO cannulation. In contrast, in our patients transported to the ECPR centre, the mean time of 000 call to EMS arrival was 8 minutes, the scene time was 41 minutes, and the transport time was 13 minutes, giving a total low flow time of 62 minutes before the start of ECMO cannulation. 
Table 2. Outcomes

\begin{tabular}{|c|c|c|c|c|c|}
\hline & ECPR centre & Non-ECPR centre & $P$ & Odds ratio* $(95 \% \mathrm{Cl})$ & $P^{*}$ \\
\hline Total number of patients & 49 & 174 & & & \\
\hline Discharged alive & $5(10.2 \%)$ & $7(4.0 \%)$ & 0.090 & $2.13(0.59-7.63)$ & 0.248 \\
\hline Discharged home & $5(100 \%)$ & $5(71.4 \%)$ & 0.470 & & \\
\hline Discharged to rehabilitation & $0(0 \%)$ & $2(28.6 \%)$ & 0.470 & & \\
\hline Alive at 1 year & $5(10.2 \%)$ & $4(2.3 \%)$ & 0.026 & $3.78(0.93-1.02)$ & 0.072 \\
\hline CPC score $1 / 2$ & $5(10.2 \%)$ & $3(1.7 \%)$ & 0.014 & $4.63(0.97-22.11)$ & 0.055 \\
\hline
\end{tabular}

ECPR = extracorporeal membrane oxygenation assisted cardiopulmonary resuscitation, CPC = Cerebral Performance Category. * After baseline adjustment.

Bartos and colleagues, ${ }^{3}$ also in Minnesota, reported the outcomes of 160 patients with an initial cardiac rhythm of $\mathrm{VF} / \mathrm{pVT}$ placed on ECMO in their receiving hospital during CPR. The median duration of cardiac arrest was 60 minutes, and neurologically favourable survival was $33 \%$.

A recent meta-analysis included 62 studies with a total of 3638 participants. ${ }^{4}$ Of these, 34 studies were of OHCA patients and 22 studies included both in-hospital and outof-hospital cardiac arrest. This review showed that 602/3366 OHCA patients (18\%) survived with favourable neurological outcomes.

There are a number of learnings from our study. First, prolonged mCPR may be associated with significant chest and/or abdominal trauma that may influence the decision in the ED to attempt ECMO cannulation. In the 49 patients at the ECPR centre, two patients had significant intra-abdominal bleeding that was only diagnosed after cannulation. The incidence of internal injury following mCPR has been previously reported..$^{13}$ In this study, post mortem findings of injuries in 113 patients having mCPR were compared with 501 patients having standard CPR. The mCPR was associated with more frequent pneumothorax (6\% v 1\%; $P=0.008)$, lung lesions $(19 \%$ $\checkmark 4 \% ; P=0.008)$, and liver lacerations (10\% $\vee 1 \%$; $P=0.001)$. Therefore, in the evaluation of suitability for $E C P R$ in the ED, bedside chest and abdominal ultrasound should be undertaken to exclude significant intrathoracic and/or intra-abdominal bleeding, which would be a contraindication to ECMO cannulation.

In addition to earlier transport of refractory arrest to an ECPR centre, there are a number of possible improvements to the current ECPR protocol in our system. First, having one ECPR centre in a very large city and the requirement to arrive at that centre between 45 and 60 minutes after onset of arrest limits the availability of ECPR to those in the inner suburban area. Consideration could be given to having staff in outer suburban and larger rural EDs trained and equipped to provide ECPR, with subsequent transfer to the ECMO centre. Alternatively, the use of a mobile ECMO vehicle to provide ECPR at a number of receiving EDs has been described. ${ }^{14}$ In that study, 58 patients with refractory OHCA were treated at their initial receiving hospital by the mobile ECMO service and $25 / 58$ patients (43\%) were discharged alive from the hospital.

ECPR at the scene has also been described in Paris, France. ${ }^{15}$ When ECPR was initiated at scene after 20 minutes of cardiac arrest, the rate of survival with good neurological function at ICU discharge or day 28 in 42 patients was $29 \%$.

In addition to decreasing the time from arrest to ECMO support, there may be other initiatives that might improve outcomes after ECPR and require further research. Firstly, the induction of therapeutic hypothermia $\left(32-34^{\circ} \mathrm{C}\right)$ may be of benefit when induced immediately after ROSC compared with delayed hypothermia or maintaining normothermia. ${ }^{16-18}$ Secondly, reperfusion with $100 \%$ oxygen may be associated with increased injury to the brain $^{19}$ and the heart ${ }^{20}$ compared with normoxic reperfusion. Thus, blending the ECMO blood flow in the ECMO circuit with oxygen plus air may improve neurological outcomes.

Our study has several limitations. First, we did not have accurate data on the time to establishment of adequate ECMO blood flow. Second, we did not have comprehensive data relating to patient interventions from the 15 non$\mathrm{ECMO}$ centres to determine the medical therapies that were administered. Also, our study was undertaken in a very large metropolitan city and our recommendations might not be applicable to smaller cities or those ambulance services without $\mathrm{mCPR}$.

\section{Conclusion}

In summary, this study found low rates of survival from refractory OHCA and a prolonged period between arrest and transport to an ECPR centre. Improved outcomes are 


\section{ORIGINAL ARTICLES}

likely if the time between arrest and ECPR is shortened. Further studies are needed to determine the optimal time to commence transport to an ECPR centre. In larger cities, additional sites with ECPR capability would also decrease the time to provide that treatment.

Acknowledgements: Dion Stub is supported by a Heart Foundation Fellowship.

\section{Competing interests}

All authors declare that they do not have any potential conflict of interest in relation to this manuscript.

\section{Author details}

Stephen A Bernard ${ }^{1,2,3}$

Sarah J Hopkins ${ }^{1,3}$

Jocasta C Ball1,3,4

Dion A Stub $1,2,3,4$

Michael W Stephenson ${ }^{1,3,5}$

Vinodh B Nanjayya ${ }^{2,3,6}$

Vincent A Pellegrino 2,6

Jayne Sheldrake ${ }^{2}$

Alexander C Richardson ${ }^{2,3,6}$

Karen L Smith ${ }^{1,3,5}$

1 Ambulance Victoria, Centre for Research and Evaluation, Melbourne, VIC, Australia.

2 Alfred Hospital, Melbourne, VIC, Australia.

3 Department of Epidemiology and Preventive Medicine, Monash University, Melbourne, VIC, Australia.

4 Baker Heart and Diabetes Institute, Melbourne, VIC, Australia.

5 Department of Paramedicine, Monash University, Melbourne, VIC, Australia.

6 Australian and New Zealand Intensive Care Society Research Centre, Melbourne, VIC, Australia.

Correspondence: steve.bernard@ambulance.vic.gov.au

doi: https://doi.org/10.51893/2022.1.OA1

\section{References}

1 Grunau B, Kime N, Leroux B, et al. Association of intra-arrest transport vs continued on-scene resuscitation with survival to hospital discharge among patients with out-of-hospital cardiac arrest. JAMA 2020; 324: 1058-67.

2 Yannopoulos D, Bartos J, Raveendran G, et al. Advanced reperfusion strategies for patients with out-of-hospital cardiac arrest and refractory ventricular fibrillation (ARREST): a phase
2 , single centre, open-label, randomised controlled trial. Lancet 2020; 396: 1807-16.

3 Bartos JA, Grunau B, Carlson C, et al. Improved survival with extracorporeal cardiopulmonary resuscitation despite progressive metabolic derangement associated with prolonged resuscitation. Circulation 2020; 141: 877-86.

4 Miraglia D, Ayala JE. Extracorporeal cardiopulmonary resuscitation for adults with shock-refractory cardiac arrest. J Am Coll Emerg Physicians Open 2021; 2: e12361.

5 Stub D, Bernard S, Pellegrino V, et al. Refractory cardiac arrest treated with mechanical CPR, Hypothermia, ECMO and Early Reperfusion (the CHEER Trial). Resuscitation 2015; 86: 88-94.

6 Leman P, Morley P. Review article: updated resuscitation guidelines for 2016: A summary of the Australian and New Zealand Committee on Resuscitation recommendations. Emerg Med Australasia 2016; 28: 379-82.

7 Ambulance Victoria. Clinical practice guidelines. https://cpg. ambulance.vic.gov.au (viewed Nov 2021).

8 Richardson ASC, Tonna JE, Nanjayya V, et al. Extracorporeal cardiopulmonary resuscitation in adults. Interim guideline consensus statement from the Extracorporeal Life Support Organization. ASAIO J 2021; 67: 221-8.

9 Bernard SA, Smith K, Finn J, et al. Induction of therapeutic hypothermia during out-of-hospital cardiac arrest using a rapid infusion of cold saline: the RINSE Trial (Rapid Infusion of Cold Normal Saline). Circulation 2016; 134: 797-805.

10 Nielsen N, Wetterslev J, Cronberg T, et al: Targeted temperature management at $33^{\circ} \mathrm{C}$ versus $36^{\circ} \mathrm{C}$ after cardiac arrest. $N$ Engl J Med 2013; 369: 2197-206.

11 Nehme Z, Bernard S, Cameron P, et al. Using a cardiac arrest registry to measure the quality of emergency medical service care: decade of findings from the Victorian Ambulance Cardiac Arrest Registry. Circ Cardiovasc Qual Outcomes 2015; 8: 56-66.

12 Smith K, Andrew E, Lijovic M, et al. Quality of life and functional outcomes 12-months after out-of-hospital cardiac arrest. Circulation 2015; 131: 174-81.

13 Ondruschka B, Baier C, Bayer R, et al. Chest compressionassociated injuries in cardiac arrest patients treated with manual chest compressions versus automated chest compression devices (LUCAS II) - a forensic autopsy-based comparison. Forensic Sci Med Pathol 2018; 14: 515-25.

14 Bartos JA, Frascone RJ, Conterato M, et al. The Minnesota mobile extracorporeal cardiopulmonary resuscitation consortium for treatment of out-of-hospital refractory ventricular fibrillation: program description, performance, and outcomes. EClinicalMedicine 2020; 29-30: 100632.

15 Lamhaut L, Hutin A, Puymirat E, et al. A pre-hospital extracorporeal cardio pulmonary resuscitation (ECPR) strategy for treatment of refractory out hospital cardiac arrest: an observational study and propensity analysis. Resuscitation 


\section{ORIGINAL ARTICLES}

2017; 117: 109-17.

16 Yamada S, Kaneko T, Kitada M, et al. Shorter interval from witnessed out-of-hospital cardiac arrest to reaching the target temperature could improve neurological outcomes after extracorporeal cardiopulmonary resuscitation with target temperature management: a retrospective analysis of a Japanese Nationwide Multicenter Observational Registry. Ther Hypothermia Temp Manag 2021; 11: 185-91.

17 Schock RB, Janata A, Peacock WF, et al. Time to cooling is associated with resuscitation outcomes. Ther Hypothermia Temp Manag 2016; 6: 208-17.
18 Dankiewicz J, Cronberg T, Lilja G, et al. Hypothermia versus normothermia after out-of-hospital cardiac arrest. $N$ Engl J Med 2021; 384: 2283-94.

19 Young PJ, Bailey M, Bellomo R, et al. Conservative or liberal oxygen therapy in adults after cardiac arrest: an individual-level patient data meta-analysis of randomised controlled trials. Resuscitation 2020; 157: 15-22.

20 Stub D, Smith K, Bernard S, et al. Air versus oxygen in patients with ST-segment elevation myocardial infarction. Circulation 2015; 131: 2143-50. 